\title{
INTEGRALITY PROPERTIES OF BÖTTCHER COORDINATES FOR ONE-DIMENSIONAL SUPERATTRACTING GERMS
}

\author{
ADRIANA SALERNO AND JOSEPH H. SILVERMAN
}

\begin{abstract}
Let $R$ be a ring of characteristic 0 with field of fractions $K$, and let $m \geq 2$. The Böttcher coordinate of a power series $\varphi(x) \in x^{m}+x^{m+1} R \llbracket x \rrbracket$ is the unique power series $f_{\varphi}(x) \in$ $x+x^{2} K \llbracket x \rrbracket$ satisfying $\varphi \circ f_{\varphi}(x)=f_{\varphi}\left(x^{m}\right)$. In this paper we study the integrality properties of the coefficients of $f_{\varphi}(x)$, partly for their intrinsic interest and partly for potential applications to $p$ adic dynamics. Results include: (1) If $p$ is prime and $R=\mathbb{Z}_{p}$ and $\varphi(x) \in x^{p}+p x^{p+1} R \llbracket x \rrbracket$, then $f_{\varphi}(x) \in R \llbracket x \rrbracket$. (2) If $\varphi(x) \in$ $x^{m}+m x^{m+1} R \llbracket x \rrbracket$, then $f_{\varphi}(x)=x \sum_{k=0}^{\infty} a_{k} x^{k} / k$ ! with all $a_{k} \in R$. (3) In (2), if $m=p^{2}$, then $a_{k} \equiv-1(\bmod p)$ for all $k$ that are powers of $p$.
\end{abstract}

\section{INTRODUCTION}

The following well-known result is essentially due to Böttcher [2].

Proposition 1. Let $K$ be a field of characteristic 0 , and let $m \geq 2$. Let

$$
\varphi(x) \in x^{m}+x^{m+1} K \llbracket x \rrbracket
$$

be a power series of the indicated form. Then there is a unique formal power series $f_{\varphi}(x) \in x+x^{2} K \llbracket x \rrbracket$ satisfying

$$
\varphi \circ f_{\varphi}(x)=f_{\varphi}\left(x^{m}\right) .
$$

There are two standard ways to prove Proposition 1. First, one can use the Böttcher equation (1) to construct a recursion that defines each coefficient of $f_{\varphi}(x)$ in terms of the earlier coefficients and the coefficients of $\varphi(x)$. Second, one can show that

$$
f_{\varphi}(x):=\lim _{n \rightarrow \infty}\left(\varphi^{\circ n}(x)\right)^{1 / m^{n}} \quad \text { converges in } K \llbracket x \rrbracket .
$$

Date: September 26, 2018.

2010 Mathematics Subject Classification. Primary: 37P10; Secondary: 11S82, $37 \mathrm{P} 20$.

Key words and phrases. formal power series, Böttcher coordinate, superattracting germ, nonarchimedean dynamics.

Silverman's research supported by Simons Collaboration Grant \#241309. 
Definition. The series $f_{\varphi}(x) \in K \llbracket x \rrbracket$ uniquely determined by (1) is called the (local) Böttcher coordinate for the series $\varphi(x)$.

For $K=\mathbb{C}$, Böttcher proved that if $\varphi(x)$ is analytic at 0 , then $f_{\varphi}(x) \in$ $\mathbb{C} \llbracket x \rrbracket$ converges on a neighborhood of 0 , and thus gives a local complex analytic conjugacy between $\varphi(x)$ and $x^{m}$. See [8, Chapter 9], for example, for a discussion of Böttcher coordinates over $\mathbb{C}$. In this paper we are interested in the convergence properties of the series $f_{\varphi}$ in the case that $K$ is a non-archimedean field, or alternatively, we want to study the integrality properties of the coefficients of the Böttcher coordinate. Suppose that $\varphi(x) \in R \llbracket x \rrbracket$ has coefficients in a ring $R$. If we further assume that the ramification degree $m$ is invertible in $R$, then the coefficients of $f_{\varphi}(x)$ are quite well-behaved, as in the following result.

Proposition 2. Let $R$ be a ring, let $m \geq 2$ be an integer satisfying $m \in$ $R^{*}$, and let

$$
\varphi(x) \in x^{m}+x^{m+1} R \llbracket x \rrbracket
$$

be a power series of the indicated form. Then both the Böttcher coordinate $f_{\varphi}(x)$ and its inverse series $f_{\varphi}^{-1}(x)$ are in $R \llbracket x \rrbracket$.

Proof. This is well-known, cf. [6. It follows easily via an induction argument similar to the proof of Theorem 3 .

The coefficients of the Böttcher coordinate become more complicated, and much more interesting, when the ramification degree $m$ is not invertible in $R$. Our first main result gives a general bound for the denominators of the coefficients of the Böttcher coordinate for maps of the form (3) without the assumption that $m$ is invertible in $R$. We also give a better bound if a few of the non-leading coefficients of $\varphi(x)$ have some additional $m$-divisibility.

Theorem 3. Let $R$ be a ring of characteristic 0 , let $m \geq 2$ be an integer, and let

$$
\varphi(x)=x^{m} \sum_{k=0}^{\infty} b_{k} x^{k} \in R \llbracket x \rrbracket \quad \text { with } b_{0}=1 .
$$

(a) Both the Böttcher coordinate $f_{\varphi}(x)$ and its inverse $f_{\varphi}^{-1}(x)$ are series of the form

$$
x \sum_{k=0}^{\infty} \frac{a_{k}}{m^{k} k !} x^{k} \quad \text { with } a_{0}=1 \text { and } a_{k} \in R \text { for all } k .
$$

(b) Suppose further that the coefficients of $\varphi$ satisfy

$$
k ! b_{k} \in m R \quad \text { for } 1 \leq k<m .
$$


For example, this is true if $\varphi(x) \in x^{m}+m x^{m+1} R \llbracket x \rrbracket$. Then the Bötcher coordinate $f_{\varphi}(x)$ and its inverse $f_{\varphi}^{-1}(x)$ are series of the form

$$
x \sum_{k=0}^{\infty} \frac{a_{k}}{k !} x^{k} \quad \text { with } a_{0}=1 \text { and } a_{k} \in R \text { for all } k .
$$

A special case of Theorem 3(b) says that if $\varphi(x) \in x^{m}+m x^{m+1} R \llbracket x \rrbracket$, then the Böttcher coordinate $f_{\varphi}(x)$ has the form $x \sum a_{k} x^{k} / k$ ! with $a_{k} \in R$. It turns out that if $m$ is prime, then we can often do much better, as shown in the following somewhat surprising result.

Theorem 4. Let $R$ be a ring of characteristic 0 with fraction field $K$, and let $p$ be a prime such that $a^{p} \equiv a(\bmod p R)$ for all $a \in R$. For example, $R$ could be $\mathbb{Z}$ or $\mathbb{Z}_{p}$. Let

$$
\varphi(x) \in x^{p}+p x^{p+1} R \llbracket x \rrbracket
$$

be a power series of the indicated form. Then the Böttcher coordinate and its inverse satisfy

$$
f_{\varphi}(x) \in R \llbracket x \rrbracket \quad \text { and } \quad f_{\varphi}^{-1}(x) \in R \llbracket x \rrbracket .
$$

We've already noted that Theorem 4, which deals with the case that $m=p$ is prime, is much stronger than Theorem 3. (b), which deals with the case that $m$ is composite. The proof of Theorem 4 relies on Fermat's little theorem, so one might suppose that Theorem 3 (b) could be strengthened by using the congruence

$$
(a+b)^{m} \cong\left(a^{p}+b^{p}\right)^{m / p} \quad(\bmod p R),
$$

which valid for $p \mid m$. However, this is not the case, as shown by the following result, whose proof Section 6 is a complicated induction on the coefficients of the Böttcher coordinate.

Theorem 5. Let $\varphi(x)=x^{p^{2}}+p^{2} x^{p^{2}+1}$, let $f_{\varphi}(x)$ be the Böttcher coordinate for $\varphi$, and write $f_{\varphi}(x)$ as

$$
f_{\varphi}(x)=x \sum_{k=0}^{\infty} \frac{a_{k}}{k !} x^{k},
$$

where $a_{k} \in \mathbb{Z}$ from Theorem $3(b)$. Then for all $k$ that are powers of $p$, we have

$$
a_{k} \equiv-1 \quad(\bmod p)
$$

Table 1 illustrates our results by giving the first few terms of the Böttcher coordinate of $\varphi(x) \in x^{m}+m x^{m+1} R \llbracket x \rrbracket$ for small values of $m$. 


\begin{tabular}{|c|l|}
\hline$m$ & Böttcher coordinate of $x^{m}+m x^{m+1}$ \\
\hline \hline 2 & $x-x^{2}+2 x^{3}-7 x^{4}+26 x^{5}-98 x^{6}+389 x^{7}-1617 x^{8}+6884 x^{9}+\cdots$ \\
\hline 3 & $x-x^{2}+3 x^{3}-12 x^{4}+52 x^{5}-246 x^{6}+1224 x^{7}-6300 x^{8}+33300 x^{9}+\cdots$ \\
\hline 4 & $x-x^{2}+\frac{7}{2} x^{3}-16 x^{4}+\frac{661}{8} x^{5}-\frac{923}{2} x^{6}+\frac{43221}{16} x^{7}-16368 x^{8}+\frac{13029155}{128} x^{9}+\cdots$ \\
\hline 5 & $x-x^{2}+4 x^{3}-21 x^{4}+125 x^{5}-801 x^{6}+5386 x^{7}-37497 x^{8}+267913 x^{9}+\cdots$ \\
\hline 6 & $x-x^{2}+\frac{9}{2} x^{3}-\frac{80}{3} x^{4}+\frac{4301}{24} x^{5}-1296 x^{6}+\frac{1416521}{144} x^{7}-\frac{695549}{9} x^{8}+\frac{79748667}{128} x^{9}+\cdots$ \\
\hline 7 & $x-x^{2}+5 x^{3}-33 x^{4}+247 x^{5}-1989 x^{6}+16807 x^{7}-146968 x^{8}+1318564 x^{9}+\cdots$ \\
\hline 8 & $x-x^{2}+\frac{11}{2} x^{3}-40 x^{4}+\frac{2639}{8} x^{5}-2926 x^{6}+\frac{435643}{16} x^{7}-262144 x^{8}+\frac{331406059}{128} x^{9}+\cdots$ \\
\hline 9 & $x-x^{2}+6 x^{3}-\frac{143}{3} x^{4}+\frac{1288}{3} x^{5}-4158 x^{6}+\frac{380120}{9} x^{7}-\frac{3994133}{9} x^{8}+4782969 x^{9}+\cdots$ \\
\hline 10 & $x-x^{2}+\frac{13}{2} x^{3}-56 x^{4}+\frac{4375}{8} x^{5}-\frac{28704}{5} x^{6}+\frac{5055273}{80} x^{7}-\frac{3596928}{5} x^{8}+\frac{5375265623}{640} x^{9}+\cdots$ \\
\hline
\end{tabular}

TABLE 1. Böttcher coordinate of $x^{m}+m x^{m+1}$

Böttcher coordinates of polynomials over $p$-adic fields have been investigated in [4, 6], where they are applied to the study of $p$-adic dynamics. (See Section 2 for details.) In this context, a key quantity is the radius of convergence of the Böttcher coordinate. Our main results yield the following estimates for this radius.

Corollary 6. Let $p$ be a prime, let $R_{p}=\left\{c \in \mathbb{C}_{p}:\|c\|_{p} \leq 1\right\}$ be the ring of integers of $\mathbb{C}_{p}$, and let $m \geq 2$ be an integer. For each indicated type of map $\varphi$, the Böttcher coordinate $f_{\varphi}$ and its inverse $f_{\varphi}^{-1}$ converge on the indicated disk $\mathcal{D}$ and define an isometry

$$
f_{\varphi}: \mathcal{D} \stackrel{\sim}{\longrightarrow} \mathcal{D} \text {. }
$$

In particular, $\varphi(x)$ is p-adically analytically conjugate to $x^{m}$ on $\mathcal{D}$.

(a) For $\varphi(x) \in x^{m}+x^{m+1} R_{p} \llbracket x \rrbracket$, as in Proposition 2 and Theorem $3(a)$, we may take

$$
\mathcal{D}= \begin{cases}\left\{x \in \mathbb{C}_{p}:\|x\|_{p}<1\right\} & \text { if } p \nmid m . \\ \left\{x \in \mathbb{C}_{p}:\|x\|_{p}<p^{-1 /(p-1)}\|m\|_{p}\right\} & \text { if } p \mid m .\end{cases}
$$

(b) For $\varphi(x)=x^{m} \sum_{k=0}^{\infty} b_{k} x^{k} / k$ ! with $b_{0}=1$ and $k ! b_{k} \in m R_{p} \llbracket x \rrbracket$ for all $1 \leq k<m$, as in Theorem 3(b), we may take

$$
\mathcal{D}=\left\{x \in \mathbb{C}_{p}:\|x\|_{p}<p^{-1 /(p-1)}\right\} .
$$

(c) For $\varphi(x) \in x^{p}+p x^{p+1} R_{p} \llbracket x \rrbracket$ as in Theorem 4, we may take

$$
\mathcal{D}=\left\{x \in \mathbb{C}_{p}:\|x\|_{p}<1\right\} .
$$

(d) For $\varphi(x)=x^{p^{2}}+p^{2} x^{p^{2}+1}$ as in Theorem 5, the radius of convergence of the Böttcher coordinate $f_{\varphi}(x)$ is exactly equal to $p^{-1 /(p-1)}$. 
Remark 7. Theorem 5 says that the $k$ 'th coefficient $a_{k} / k$ ! of the Böttcher coordinate of $x^{p^{2}}+p^{2} x^{p^{2}+1}$ satisfies $a_{k} \equiv-1(\bmod p)$ provided that $k$ is a power of $p$. Experiments suggest that this reflects a much more widespread phenomenon. For example, we suspect that if $k$ is a multiple of $p$, then we always have

$$
a_{k} \equiv(-1)^{k / p}(\bmod p) \text {. }
$$

In Section 8 we assemble a number of conjectures, based on numerical evidence, that describe various $p$-adic properties of the Böttcher coordinate of $x^{p^{2}}+p^{r+2} x^{p^{2}+1}$. In particular, we conjecture that the radius of convergence of the Böttcher coordinate is exactly $p^{-p^{-r} /(p-1)}$.

Remark 8. Theorem 4 tells us that if $t \in \mathbb{Z}_{p}$, then the Böttcher coordinate of $\varphi(x)=x^{p}+p t x^{p+1}$ has $p$-integral coefficients. However, if we treat $t$ as an indeterminate, then the coefficients of $f_{\varphi}(x)$ are in $\mathbb{Q}[t]$, but they often fail to be in $\mathbb{Z}[t]$. For example, for $p=2$ we find that

$$
f_{\varphi}(x)=x-t x^{2}+\left(\frac{5 t^{2}-t}{2}\right) x^{3}-\left(8 t^{3}-t^{2}\right) x^{4}+\left(\frac{231 t^{4}-30 t^{3}+9 t^{2}-2 t}{8}\right) x^{5}+\cdots,
$$

and for $p=3$ we have

$$
f_{\varphi}(x)=x-t x^{2}+3 t^{2} x^{3}-\left(\frac{35 t^{3}+t}{3}\right) x^{4}+\left(\frac{154 t^{4}+2 t^{2}}{3}\right) x^{5}-\left(243 t^{5}+3 t^{3}\right) x^{6}+\cdots .
$$

Although the coefficients of $f_{\varphi}(x)$ are in $\mathbb{Q}[t]$, we can verify that they are integer-valued, as they must be according to Theorem 4 , by writing their Newton-Mahler expansions. For example, for $p=2$ the coefficient of $x^{5}$ in $f_{\varphi}(x)$ has Newton-Mahler expansion

$$
\frac{231 t^{4}-30 t^{3}+9 t^{2}-2 t}{8}=693\left(\begin{array}{l}
t \\
4
\end{array}\right)+1017\left(\begin{array}{l}
t \\
3
\end{array}\right)+384\left(\begin{array}{l}
t \\
2
\end{array}\right)+26\left(\begin{array}{l}
t \\
1
\end{array}\right) \text {. }
$$

Remark 9. In this paper we start with a power series $\varphi(x)=x^{m}+\cdots$ having a critical point at 0 and study the arithmetic properties of the coefficients of the Böttcher coordinate $f_{\varphi}(x)$ that conjugates $\varphi(x)$ to $x^{m}$. We mention that if instead we start with an invertible power series $f(x)=x+\cdots$, then there is a unique power series for which $f$ is the $m$-power Böttcher coordinate. Indeed, replacing $x$ by $f^{-1}(x)$ in the Böttcher equation (11) yields

$$
\varphi(x)=f\left(f^{-1}(x)^{m}\right),
$$

and this $\varphi$ clearly satisfies $\varphi(x)=x^{m}+\cdots$ and $f_{\varphi}(x)=\varphi(x)$.

We briefly indicate the contents of this paper. In Section 2 we review some of the earlier work that has been done on Böttcher coordinates in the $p$-adic and characteristic $p$ setting, after which Section 3 contains some useful facts concerning inverses of various types of power series. 
This is followed in Sections 4, 5, and 5 with the proofs, respectively, of Theorems 4, 3 and 5. In Section 7 we use our earlier results to prove Corollary 6. Finally, in Section 8 we give various precise conjectures describing the coefficients of the Böttcher coordinate for maps of the form $x^{p^{2}}+p^{r+2} x^{p^{2}+1}$.

\section{EARlier and Related Work}

In this section we briefly summarize earlier work on $p$-adic and characteristic $p$ Böttcher coordinates and relate it to the present paper. Böttcher coordinates of polynomials over $p$-adic fields appear to have first been studied by Ingram [6] in the case that the ramification degree $m$ is relatively prime to $p$. This work was extended and generalized by DeMarco, Ghioca, Krieger, Nguyen, Tucker, and Ye [4] in two ways. First, they allow $m$ to be divisible by $p$, and second, they work uniformly in families of polynomials. Both of these earlier papers consider only the Böttcher coordinate of a monic polynomial in a neighborhood of $\infty$, i.e., they restrict attention to rational functions having a totally ramified fixed point. This contrasts with our results, which apply in particular to rational functions having a critical fixed point that need not be totally ramified.

We state the result of DeMarco et al., which generalizes Ingram [6, Theorem 2], but we conjugate by $x \rightarrow x^{-1}$ so as to move their (totally) ramified fixed point to 0 .

Theorem 10 (DeMarco et al. [4, Theorem 6.5]). Let $m \geq 2$, let $\beta_{1}, \ldots, \beta_{m} \in \mathbb{C}_{p}$, let $\|\cdot\|_{p}$ be the usual absolute value on $\mathbb{C}_{p}$ normalized so that $\|p\|_{p}=p^{-1}$, and let $\varphi(x) \in \mathbb{C}_{p} \llbracket x \rrbracket$ be the Taylor series around 0 of the rational function

$$
\frac{x^{m}}{1+\beta_{1} x+\beta_{2} x^{2}+\cdots+\beta_{m} x^{m}} \in \mathbb{C}_{p}[x] .
$$

Set

$$
\|\boldsymbol{\beta}\|_{p}:=\max \left\{1,\left\|\beta_{1}\right\|_{p}, \ldots,\left\|\beta_{m}\right\|_{p}\right\}
$$

and let $\mathcal{D}_{\varphi}$ be the disk

$$
\mathcal{D}_{\varphi}:= \begin{cases}\left\{x \in \mathbb{C}_{p}:\|x\|_{p}<\|\boldsymbol{\beta}\|_{p}^{-1}\right\} & \text { if } p \nmid m, \\ \left\{x \in \mathbb{C}_{p}:\|x\|_{p}<p^{-1 /(p-1)}\|m\|_{p}\|\boldsymbol{\beta}\|_{p}^{-1}\right\} & \text { if } p \mid m .\end{cases}
$$

Then the Böttcher coordinate $f_{\varphi}(x)$ converges on $\mathcal{D}$ and defines an injective map $f_{\varphi}: \mathcal{D} \hookrightarrow \mathbb{C}_{p}$.

We observe that if we apply Theorem 10 in the case that $\beta_{1}, \ldots, \beta_{m}$ are $p$-integral, then $\varphi(x)$ has $p$-integral coefficients, and the convergence results in Theorem 10 are the same as those obtained in Corollary 6(a). 
However, even if we assume that $\beta_{1}, \ldots, \beta_{m}$ are highly $p$-divisible in Theorem 10, we obtain no improvement in the disk $\mathcal{D}$, since we always have $\|\boldsymbol{\beta}\|_{p} \geq 1$. In particular, Theorem 10 does not imply the stronger convergence estimates given in Corollary 6(b,c). On the other hand, the results of Ingram and of DeMarco et al. do apply to series whose coefficients are not necessarily $p$-integral, a situation that we do not consider in the present paper. Roughly speaking, the earlier papers include (polynomial) maps having bad reduction, while our results deal with maps having good reduction, and we give improved estimates in the case that $\varphi(x) \equiv x^{m}(\bmod m)$, which one might say is the case that $\varphi(x)$ has "very good reduction."

We note again that the work of Ingram [6] and DeMarco et al [4] deal only with the Böttcher coordinates of polynomial functions, i.e., rational functions having a totally ramified fixed point. This contrasts with our results, which apply in particular to rational functions having a critical fixed point that need not be totally ramified, although we expect that their arguments could be adapted to the more general setting.

We also note that these earlier papers construct the Böttcher coordinate via the classical limit (2) mentioned in the introduction. This may well have some technical advantages, but it seems that in order to study subtler $p$-adic properties of Böttcher coordinates, it is necessary to use the finer combinatorial information provided by the recursive formula for the coefficients of the Böttcher coordinate, as is done in the present paper.

Remark 11. Superattracting germs in characteristic $p$ present many additional complications if the ramification index is divisible by $p$. In particular, the Böttcher coordinate need not exist, and one obtains interesting parameter and moduli spaces of Böttcher-like coordinates. The case $\varphi(x) \in x^{p}+x^{p+1} K \llbracket x \rrbracket$ was studied by Spencer in his thesis [12], and the general case was investigated by Ruggiero in [10].

Remark 12. Böttcher coordinates have also been studied for higher dimensional maps $\mathbb{C}^{N} \rightarrow \mathbb{C}^{N}$; see for example [3]. It would be interesting to investigate the higher dimensional situation for non-archimedean fields, but we do not do so in the present paper.

Remark 13. There is, of course, a wide body of work on linearization of maps at non-critical fixed points, i.e., at fixed points that are not superattracting, in both the complex and the non-archimedean settings. For the latter, see for example [1, 5]. 


\section{INVERSES OF POWER SERIES}

In this section we describe various sets of power series that are invariant under taking inverses. We will use the following well-known formula for the $k$ 'th derivative of the composition of functions.

Lemma 14 (Formula of Faá di Bruno and Arbogast). Let $F(x)$ and $G(x)$ be functions that are sufficiently differentiable. Then the $k$ 'th derivative of the composition $F \circ G$ is given by the formula

$$
\begin{array}{r}
D_{x}^{k} F(G(x))=\sum_{1 \cdot e_{1}+2 \cdot e_{2}+3 \cdot e_{3}+\cdots+k \cdot e_{k}=k} \frac{k !}{e_{1} ! 1 !^{e_{1}} e_{2} ! 2 !^{e_{2}} \cdots e_{k} ! k !^{! e_{k}}} \\
\cdot\left(D_{x}^{e_{1}+\cdots+e_{k}} F\right)(G(x)) \cdot \prod_{j=1}^{k}\left(D_{x}^{j} G(x)\right)^{e_{j}} .
\end{array}
$$

Proof. See [7, 9], for example, for proofs of this formula, which dates to the 19 'th century.

Parts (a) and (b) of the next proposition are well-known results, but (c) and (d), which are essentially equivalent one another, are less so. In particular, we do not see an easy way to use (b), or its usual proof by induction as in [11, Lemma IV.5.4], to prove (c).

Proposition 15. Let $R$ be a ring of characteristic 0 . Then each of the following sets of power series $\mathcal{P}_{1}(R), \ldots, \mathcal{P}_{4}(R)$ in $R \llbracket x \rrbracket$ satisfies

$$
f(x) \in \mathcal{P}_{i}(R) \quad \Longleftrightarrow \quad f^{-1}(x) \in \mathcal{P}_{i}(R) .
$$

(a) $\mathcal{P}_{1}(R):=\left\{x \sum_{k=0}^{\infty} a_{k} x^{k}: a_{0}=1\right.$ and $\left.a_{1}, a_{2}, \ldots \in R\right\}$.

(b) $\mathcal{P}_{2}(R):=\left\{x \sum_{k=0}^{\infty} \frac{a_{k} x^{k}}{(k+1) !}: a_{0}=1\right.$ and $\left.a_{1}, a_{2}, \ldots \in R\right\}$.

(c) $\mathcal{P}_{3}(R):=\left\{x \sum_{k=0}^{\infty} \frac{a_{k} x^{k}}{k !}: a_{0}=1\right.$ and $\left.a_{1}, a_{2}, \ldots \in R\right\}$.

(d) $\mathcal{P}_{4}(R):=\left\{x \sum_{k=0}^{\infty} \frac{a_{k} x^{k}}{m^{k} k !}: a_{0}=1\right.$ and $\left.a_{1}, a_{2}, \ldots \in R\right\}$.

Proof. (a) After reindexing, this is [11, Lemma IV.2.4].

(b) Again after reindexing, this is [11, Lemma IV.5.4].

(c) As noted earlier, we do not see a way to use (b) to prove (c), so we give a direct proof. We let $K$ be the fraction field of $R$, and we set the 
notation

$$
f(x)=x \sum_{k=0}^{\infty} \frac{a_{k} x^{k}}{k !} \in \mathcal{P}_{3}(R) \text { and } g(x):=f^{-1}(x)=x \sum_{k=0}^{\infty} \frac{b_{k} x^{k}}{k !} \in K \llbracket x \rrbracket .
$$

The facts that $f(g(x))=x$ and $a_{0}=1$ imply that $b_{0}=1$, so it remains to prove that every $b_{k}$ is in $R$.

We set some useful notation. For any list $\boldsymbol{e}=\left(e_{1}, e_{2}, \ldots\right)$ of nonnegative integers, with only finitely many non-zero entries, we let

$$
\begin{aligned}
& \sigma(\boldsymbol{e})=e_{1}+e_{2}+e_{3}+\cdots, \\
& \nu(\boldsymbol{e})=1 \cdot e_{1}+2 \cdot e_{2}+3 \cdot e_{3}+\cdots .
\end{aligned}
$$

We apply the formula of Faá di Bruno (Lemma 14) to compute the $n$ 'th derivative of the composition $f(g(x))$ and evaluate at 0 , where we note that with our labeling, we have

$$
D_{x}^{k} f(0)=k a_{k-1} \quad \text { and } \quad D_{x}^{k} g(0)=k b_{k-1} .
$$

This yields

$$
\begin{aligned}
D_{x}^{n}(f \circ g)(0) & =\sum_{\nu(\boldsymbol{e})=n} n ! \prod_{j=1}^{n} \frac{1}{j !^{e_{j}} \cdot e_{j} !} \cdot\left(D_{x}^{\sigma(\boldsymbol{e})} f\right)(g(0)) \cdot \prod_{j=1}^{n}\left(D_{x}^{j} g(0)\right)^{e_{j}} \\
& =\sum_{\nu(\boldsymbol{e})=n} n ! \prod_{j=1}^{n} \frac{1}{j !^{e_{j}} \cdot e_{j} !} \cdot \sigma(\boldsymbol{e}) a_{\sigma(\boldsymbol{e})-1} \prod_{j=1}^{n}\left(j b_{j-1}\right)^{e_{j}}
\end{aligned}
$$

On the other hand, since $f(g(x))=x$, we have $D_{x}^{n}(f \circ g)=0$ for all $n \geq 2$. This yields a recursion for the coefficients of $g(x)$.

Thus the term with $\nu(\boldsymbol{e})=n$ and $\sigma(\boldsymbol{e})=1$, i.e., the term with $e_{n}=1$ and all other $e_{j}=0$, is

$$
n ! \cdot \frac{1}{n !^{1} \cdot 1 !} \cdot 1 \cdot a_{0} \cdot n b_{n-1}=n b_{n-1},
$$

so if we assume (by induction) that $b_{1}, \ldots, b_{n-2} \in R$, and use $a_{0}=b_{0}=$ 1 and $a_{k} \in R$ for all $k$, we see that in order to prove that $b_{n-1} \in R$, it suffices to show that for every $\boldsymbol{e}$ satisfying $\nu(\boldsymbol{e})=n$, we have

$$
n ! \prod_{j=1}^{n} \frac{1}{j ! e_{j} \cdot e_{j} !} \cdot \sigma(\boldsymbol{e}) \cdot \prod_{j=1}^{n} j^{e_{j}} \equiv 0 \quad(\bmod n) .
$$

The validity of this congruence, which is by no means obvious, is proven in a separate lemma at the end of this section; see Lemma 16.

(d) This follows from (c). Thus let $f(x)=x \sum a_{k} x^{k} / m^{k} k ! \in \mathcal{P}_{4}(R)$. Then $F(x):=x \sum a_{k} x^{k} / k ! \in \mathcal{P}_{3}(R)$, so (c) tells us hat $F^{-1}(x)=$ 
$x \sum b_{k} x^{k} / k$ ! with $b_{0}=1$ and all $b_{k} \in R$. Using the identity $f(x)=$ $m F(x / m)$, we see that

$$
f^{-1}(x)=m F^{-1}(x / m)=m \cdot \frac{x}{m} \sum_{k=0}^{\infty} \frac{b_{k}(x / m)^{k}}{k !}=x \sum_{k=0}^{\infty} \frac{b_{k} x^{k}}{m^{k} k !},
$$

which proves that $f^{-1}(x) \in \mathcal{P}_{4}(R)$.

Lemma 16. Let $e_{1}, e_{2}, \ldots, e_{n}$ be non-negative integers satisfying

$$
1 \cdot e_{1}+2 \cdot e_{2}+\cdots+n \cdot e_{n}=n .
$$

Then

$$
n ! \prod_{j=1}^{n} \frac{1}{j ! e_{j} \cdot e_{j} !} \cdot\left(e_{1}+e_{2}+\cdots+e_{n}\right) \cdot \prod_{j=1}^{n} j^{e_{j}} \equiv 0 \quad(\bmod n) .
$$

Proof. To ease notation, we let

$$
m=e_{1}+e_{2}+\cdots+e_{n} .
$$

For the given list $\boldsymbol{e}=\left(e_{1}, \ldots, e_{n}\right)$, let $Z(\boldsymbol{e})$ be the collection of all expressions of the form

$$
\left(\left(S_{1}, s_{1}\right),\left\{\left(S_{2}, s_{2}\right), \ldots,\left(S_{m}, s_{m}\right)\right\}\right)
$$

where:

(i) $S_{1}, \ldots, S_{m}$ are disjoint subsets of $\mathbb{Z} / n \mathbb{Z}$.

(ii) For each $1 \leq i \leq m$ we have $s_{i} \in S_{i}$.

(iii) For each $1 \leq k \leq n$ we have $e_{k}=\#\left\{1 \leq i \leq m: \# S_{i}=k\right\}$.

We observe that (i) and (iii) imply that $\mathbb{Z} / n \mathbb{Z}$ is equal to the disjoint union of $S_{1}, \ldots, S_{m}$. We also note that an element of $Z(\boldsymbol{e})$ consists of a distinguished pointed set $\left(S_{1}, s_{1}\right)$ and an unordered collection of $m-1$ additional pointed sets $\left(S_{2}, s_{2}\right), \ldots,\left(S_{m}, s_{m}\right)$.

We claim that

$$
\# Z(\boldsymbol{e})=n ! \prod_{j=1}^{n} \frac{1}{j !^{e_{j}} \cdot e_{j} !} \cdot m \cdot \prod_{j=1}^{n} j^{e_{j}},
$$

i.e., we claim that $\# Z(\boldsymbol{e})$ is exactly the quantity that we are trying to prove is divisible by $n$. To see this, we count the number of ways to create an element of $Z(\boldsymbol{e})$. First we take $\mathbb{Z} / n \mathbb{Z}$ and partition it into $m$ subsets $S_{1}, \ldots, S_{m}$ consisting of $e_{1}$ subsets containing 1 element, $e_{2}$ subsets containing 2 elements, etc. The number of ways to do this if we keep track of the order of $S_{1}, \ldots, S_{m}$ is the multinomial coefficient

$$
\left(\begin{array}{c}
n \\
1,1, \ldots, 1,2,2, \ldots, 2, \ldots
\end{array}\right)=\frac{n !}{1 !^{e_{1}} \cdot 2 !^{e_{2}} \cdots n !^{e_{n}}} .
$$


However, if we don't care about the order, then we need to divide by $e_{1}$ ! to account for reordering the 1-element subsets and by $e_{2}$ ! to account for reordering the 2-element subsets, etc. This accounts for the factor of $\prod e_{j}$ ! in the denominator of (5). Next, we actually want $S_{1}, \ldots, S_{m}$ to be pointed subsets, so we choose one element from each $S_{i}$. This can be done in $\prod \# S_{i}=\prod j^{e_{j}}$ ways, where the equality follow from (iii). Finally we need to choose one of the pointed sets $\left(S_{i}, s_{i}\right)$ to be distinguished, which can be done in $m$ ways. This concludes the verification of (5]).

For any subset $S \subset \mathbb{Z} / n \mathbb{Z}$, we let

$$
S+1:=\{s+1 \bmod n: s \in S\} .
$$

We define an "increment-by-1" operation on $Z(\boldsymbol{e})$ by the formula

$$
\begin{gathered}
I: Z(\boldsymbol{e}) \stackrel{\sim}{\longrightarrow} Z(\boldsymbol{e}), \\
I\left(\left(S_{1}, s_{1}\right),\left\{\left(S_{2}, s_{2}\right), \cdots,\left(S_{m}, s_{m}\right)\right\}\right) \\
=\left(\left(S_{1}+1, s_{1}+1\right),\left\{\left(S_{2}+1, s_{2}+1\right), \cdots,\left(S_{m}+1, s_{m}+1\right)\right\}\right) .
\end{gathered}
$$

Now the key observation, for which we thank Melody Chan, is that the increase-by-1 operator is a permutation of the set $Z(\boldsymbol{e})$ for which the orbit of every element has size exactly $n$. This will obviously imply that $n$ divides $\# Z(\boldsymbol{e})$, so it remains to prove that truth of this observation.

Since we are working in $\mathbb{Z} / n \mathbb{Z}$, it is clear that the $n$ 'th iterate $I^{n}$ acts as the identity map on $Z(\boldsymbol{e})$, so it suffices to prove that if $I^{k}$ fixes an element

$$
\left(\left(S_{1}, s_{1}\right),\left\{\left(S_{2}, s_{2}\right), \cdots,\left(S_{m}, s_{m}\right)\right\}\right) \in Z(\boldsymbol{e}),
$$

then $n \mid k$. But if $I^{k}$ fixes this element, then incrementing by $k$ must fix the distinguished point $s_{1}$ of the distinguished pointed subset $\left(S_{1}, s_{1}\right)$, i.e.,

$$
s_{1} \equiv s_{1}+k(\bmod n) \text {. }
$$

Hence $n \mid k$, which completes the proof of Lemma 16.

Question 17. Continuing with the notation in Lemma 16, if $e_{1}<n$, is it true that we always have

$$
n ! \prod_{j=1}^{n} \frac{1}{j ! e^{e_{j}} \cdot e_{j} !} \cdot \prod_{j=1}^{n} j^{e_{j}} \equiv 0 \quad(\bmod n) ?
$$

We have verified this stronger result experimentally for various values of $n$ and $\boldsymbol{e}$. 


\section{Proof of Theorem 4}

We start by setting some notation for truncating and for picking out coefficients of power series. This notation will be used in this section and in Section 6. For any power series $P(x)=\sum_{k \geq 0} c_{k} x^{k}$, we write

$$
P^{[k]}(x)=\sum_{i=0}^{k} c_{i} x^{i} \quad \text { and } \quad P(x)\left[x^{k}\right]=c_{k} .
$$

Proof of Theorem 4. Write

$$
\varphi(x)=x^{p}(1+p \psi(x)) \quad \text { with } \psi(x) \in x R \llbracket x \rrbracket .
$$

We assume that $f_{\varphi}^{[k]}(x) \in R[x]$, and we proceed to prove that $f_{\varphi}^{[k+1]}(x) \in$ $R[x]$. To ease notation, we write

$$
f(x)=f_{\varphi}^{[k]}(x)+\beta x^{k+1}+\cdots \quad \text { with } \beta=a_{k+1} .
$$

Then $\beta$ is determined by its appearance in the $x^{p+k}$ coefficient of the defining relation (11). Thus

$$
\begin{aligned}
& 0\left.=\left(\varphi\left(f_{\varphi}^{[k]}(x)+\beta x^{k+1}\right)-f_{\varphi}^{[k]}\left(x^{p}\right)\right)\left[x^{p+k}\right] \quad \text { using (1) }\right) \\
&=\left(\left(f_{\varphi}^{[k]}(x)+\beta x^{k+1}\right)^{p}\left(1+p \psi\left(f_{\varphi}^{[k]}(x)+\beta x^{k+1}\right)\right)-f_{\varphi}^{[k]}\left(x^{p}\right)\right)\left[x^{p+k}\right] \\
&\text { using (17) }) \\
&=\left(\left(f_{\varphi}^{[k]}(x)^{p}+p \beta f_{\varphi}^{[k]}(x)^{p-1} x^{k+1}\right)\left(1+p \psi\left(f_{\varphi}^{[k]}(x)+\beta x^{k+1}\right)\right)\right. \\
&\left.\quad-f_{\varphi}^{[k]}\left(x^{p}\right)\right)\left[x^{p+k}\right] \\
&=\left(\left(f_{\varphi}^{[k]}(x)^{p}+p \beta x^{p+k}\right)\left(1+p \psi\left(f_{\varphi}^{[k]}(x)+\beta x^{k+1}\right)\right)-f_{\varphi}^{[k]}\left(x^{p}\right)\right)\left[x^{p+k}\right] \\
& \quad \operatorname{since} f_{\varphi}^{[k]}(x) \in x+x_{\varphi}^{[k]}(x) \in x[x], \\
&=\left(\left(f_{\varphi}^{[k]}(x)^{p}+p \beta x^{p+k}\right)\left(1+p \psi\left(f_{\varphi}^{[k]}(x)\right)\right)-f_{\varphi}^{[k]}\left(x^{p}\right)\right)\left[x^{p+k}\right] \\
& \quad \operatorname{since}\left(f_{\varphi}^{[k]}(x)^{p}+p \beta x^{p+k}\right) \in x^{p} R[x] \text { and } \psi(x) \in x R[x], \\
&=\left(f_{\varphi}^{[k]}(x)^{p}+p \beta x^{p+k}+f_{\varphi}^{[k]}(x)^{p} p \psi\left(f_{\varphi}^{[k]}(x)\right)-f_{\varphi}^{[k]}\left(x^{p}\right)\right)\left[x^{p+k}\right] \\
& \quad \operatorname{since~} \psi\left(f_{\varphi}^{[k]}(x)\right) \in x R[x] .
\end{aligned}
$$

Hence

$$
\beta=a_{k+1}=\frac{1}{p}\left(f_{\varphi}^{[k]}\left(x^{p}\right)-f_{\varphi}^{[k]}(x)^{p}\right)\left[x^{p+k}\right]-\left(f_{\varphi}^{[k]}(x)^{p} \psi\left(f_{\varphi}^{[k]}(x)\right)\right)\left[x^{p+k}\right] .
$$


The assumptions that $f_{\varphi}^{[k]}$ and $\psi(x)$ have coefficients in $R$ imply that the second term has coefficients in $R$. As for the first term, it has coefficients in $R$, since for any polynomial $F(x) \in R[x]$, we have

$$
F(x)^{p} \equiv F\left(x^{p}\right) \quad(\bmod p R[x]) .
$$

We note that (8) is the property that requires the assumption that $a^{p} \equiv a(\bmod p R)$ for every $a \in R$. Without that assumption, we would only have $F(x)^{p} \equiv \tilde{F}\left(x^{p}\right)(\bmod p R[x])$, where $\tilde{F}$ is obtained from $F$ by raising its coefficients to the $p$ th power. This completes the proof of Theorem 4 .

\section{Proof of Theorem 3}

We start with an elementary, but useful, description of the $k$ 'th derivative of the $m$ 'th power of a function. For notational convenience, we let $D_{x}$ denote differentiation with respect to $x$. In particular, we note that $D_{x}$ operates formally on power series rings such as $K \llbracket x \rrbracket$.

Definition. For integers $m \geq 1$ and $k \geq 1$, define a submodule of the graded polynomial ring $\mathbb{Z}\left[T_{0}, T_{1}, T_{2}, \ldots, T_{r}\right]$ by

$$
\begin{aligned}
\mathbb{Z}\left[T_{0}, T_{1}, T_{2}, \ldots, T_{r}\right]^{[m, k]} & \\
& :=\operatorname{Span}_{\mathbb{Z}}\left\{T_{0}^{e_{0}} T_{1}^{e_{1}} T_{2}^{e_{2}} \cdots T_{r}^{e_{r}}: \sum_{\ell=0}^{r} e_{\ell}=m \text { and } \sum_{\ell=0}^{r} \ell e_{\ell}=k\right\} .
\end{aligned}
$$

In other words, $\mathbb{Z}\left[T_{0}, \ldots, T_{r}\right]^{[m, k]}$ is the span of the monomials of degree $m$ and weight $k$, according to the grading wt $\left(T_{\ell}\right)=\ell$.

Lemma 18. Let $y$ be a sufficiently differentiable function of $x$, let $k \geq 1$, and let $m \geq 1$. There is a polynomial $\Delta_{m, k} \in \mathbb{Z}\left[T_{0}, \ldots, T_{k-1}\right]^{[m, k]}$ such that

$$
D_{x}^{k}\left(y^{m}\right)=m y^{m-1} D_{x}^{k}(y)+m \Delta_{m, k}\left(y, D_{x} y, D_{x}^{2} y, \ldots, D_{x}^{k-1} y\right) .
$$

Proof. We induct on $k$. For $k=1$ this is just the chain rule $D_{x}\left(y^{m}\right)=$ $m y^{m-1} D_{x}(y)$, so $\Delta_{m, 1}=0$. Assume true for $k$. We observe that

$$
D_{x}\left(m y^{m-1} D_{x}^{k}(y)\right)=m y^{m-1} D_{x}^{k+1}(0)+m(m-1) y^{m-2} D_{x}(y) D_{x}^{k}(y),
$$

while differentiating a monomial of degree $m$ and weight $k$ yields a sum of monomials that have degree $m$ and weight $k+1$. Hence $D_{x}^{k+1}\left(y^{m}\right)$ has the desired form.

Remark 19. We note that Lemma 18 may also be derived as a special case of the classical formula of Faá di Bruno for the $k$ 'the derivative of a composition of functions. Thus setting $F(x)=x^{m}$, with $G(x)=y$ 
in Lemma 14, we see that the term in (4) with $e_{k}=1$, and necessarily $e_{1}=\cdots=e_{k-1}=0$, is

$$
\frac{k !}{e_{k} !(k !)^{e_{k}}}\left(D_{x} F\right)(y) \cdot\left(D_{x}^{k}(y)\right)^{e_{k}}=m y^{m-1} D_{x}^{k}(y)
$$

matching the initial term in Lemma 18. And the other terms have the desired form once we note that for any $\ell \geq 1$, the term

$$
\left(D_{x}^{\ell} F\right)(y)=m(m-1) \cdots(m-\ell+1) y^{m-\ell}
$$

has the desired factor of $m$, and that the quantity $\left(D_{x}^{j} y\right) / j$ ! is integral.

We are now ready to prove Theorem 3

Proof of Theorem 3. It turns out to be somewhat easier to work with the inverse Böttcher coordinate, so we let $F(x)=f_{\varphi}^{-1}(x) \in K \llbracket x \rrbracket$. Thus $F$ is determined by the functional equation

$$
F(\varphi(x))=F(x)^{m}
$$

We define series $\nu(x)$ and $G(x)$ by

$$
\varphi(x)=x^{m} \nu(x)=x^{m} \sum_{i=0}^{\infty} b_{i} x^{i} \quad \text { and } \quad F(x)=x G(x)=x \sum_{\ell=0}^{\infty} \frac{c_{\ell}}{\ell !} x^{\ell} .
$$

Substituting these expressions for $F$ and $\varphi$ into (9) and canceling $x^{m}$ yields

$$
\nu(x) G\left(x^{m} \nu(x)\right)=G(x)^{m} .
$$

This functional equation determines the coefficients of $G(x)$ in terms of the coefficients of $\nu(x)$.

We are going to take the $k^{\prime}$ th derivative of both sides of (10) and evaluate at $x=0$. For the right-hand side we use Lemma 18 with $y=G(x)$. Noting that $\left(D_{x}^{\ell} G\right)(0)=c_{\ell}$ and $c_{0}=1$, we obtain

$$
\left.D_{x}^{k}\left(G(x)^{m}\right)\right|_{x=0}=m c_{k}+m \Delta_{m, k}\left(c_{0}, c_{1}, \ldots, c_{k-1}\right),
$$

where $\Delta_{m, k}\left(T_{0}, \ldots, T_{k-1}\right) \in \mathbb{Z}\left[T_{0}, \ldots, T_{k-1}\right]^{[m, k]}$ is as defined in Lemma 18 . 
In order to handle the left-hand side of (10), we expand $G\left(x^{m} \nu(x)\right)$ as a series and differentiate. Thus

$$
\begin{aligned}
D_{x}^{k}\left(\nu(x) G\left(x^{m} \nu(x)\right)\right) & =D_{x}^{k}\left(\nu(x) \sum_{\ell=0}^{\infty} \frac{c_{\ell}}{\ell !}\left(x^{m} \nu(x)\right)^{\ell}\right) \\
& =\sum_{\ell=0}^{\infty} \frac{c_{\ell}}{\ell !} D_{x}^{k}\left(x^{m \ell} \nu(x)^{\ell+1}\right) \\
& =\sum_{\ell=0}^{\infty} \frac{c_{\ell}}{\ell !} \sum_{i+j=k}\left(\begin{array}{c}
k \\
i
\end{array}\right) D_{x}^{i}\left(x^{m \ell}\right) \cdot D_{x}^{j}\left(\nu(x)^{\ell+1}\right) .
\end{aligned}
$$

Evaluating at $x=0$, we note that

$$
\left.D_{x}^{i}\left(x^{m \ell}\right)\right|_{x=0}= \begin{cases}(m \ell) ! & \text { if } i=m \ell \\ 0 & \text { otherwise }\end{cases}
$$

so the only term that remains in the inner sum is $(i, j)=(m \ell, k-m \ell)$. Further, since $0 \leq j \leq k$, this term appears only if $\ell \leq k / m$. Hence

$$
\left.D_{x}^{k}\left(\nu(x) G\left(x^{m} \nu(x)\right)\right)\right|_{x=0}=\left.\sum_{\ell=0}^{\lfloor k / m\rfloor} \frac{c_{\ell}}{\ell !} \cdot\left(\begin{array}{c}
k \\
m \ell
\end{array}\right)(m \ell) ! \cdot D_{x}^{k-m \ell}\left(\nu(x)^{\ell+1}\right)\right|_{x=0} .
$$

We next observe that

$$
\frac{(m \ell) !}{m ! \ell !} \in \mathbb{Z} \quad \text { for } \ell \geq 1
$$

so pulling off the $\ell=0$ term and using the fact that $c_{0}=1$, we find that

$$
\left.D_{x}^{k}\left(\nu(x) G\left(x^{m} \nu(x)\right)\right)\right|_{x=0} \in k ! b_{k}+\sum_{\ell=1}^{\lfloor k / m\rfloor} m ! \cdot c_{\ell} \cdot \mathbb{Z}\left[b_{1}, b_{2}, \ldots\right] .
$$

Subsituting (11) and (12) into the $k$ 'th derivative of (10) evaluated at $x=0$, and dividing by $m$, we obtain

$$
c_{k} \in-\Delta_{m, k}\left(c_{0}, c_{1}, \ldots, c_{k-1}\right)+\frac{k ! b_{k}}{m}+\sum_{\ell=1}^{\lfloor k / m\rfloor} c_{\ell} \cdot \mathbb{Z}\left[b_{1}, b_{2}, \ldots\right] .
$$

We now proceed by induction, starting from $c_{0}=1$. If we assume that $k ! b_{k} \in m R$, as in part (b), then it is clear from (13) that

$$
c_{0}, c_{1}, \ldots, c_{k-1} \in R \Longrightarrow c_{k} \in R \text {. }
$$

Hence under the assumption in (b), we see that $f_{\varphi}^{-1}(x)=\sum c_{k} x^{k} / k$ ! with $c_{k} \in R$.

In order to prove (a), our induction hypothesis is that $c_{\ell} \in m^{-\ell} R$ for all $0 \leq \ell<k$, and our goal is to conclude that $c_{k} \in m^{-k} R$. We 
consider the integrality properties of each of the terms in (13). First, the term $\Delta_{m, k}\left(c_{0}, \ldots, c_{k-1}\right)$ is a $\mathbb{Z}$-linear combination of monomials of the form

$$
c_{0}^{e_{0}} c_{1}^{e_{1}} \cdots c_{k-1}^{e_{k-1}} \quad \text { with } \quad \sum_{\ell=0}^{k-1} \ell e_{\ell}=k .
$$

Writing $c_{\ell}=\gamma_{\ell} / m^{\ell}$ with $\gamma_{\ell} \in R$, we see that

$$
c_{0}^{e_{0}} c_{1}^{e_{1}} \cdots c_{k-1}^{e_{k-1}}=\frac{\gamma_{0}^{e_{0}} \gamma_{1}^{e_{1}} \cdots \gamma_{k-1}^{e_{k-1}}}{m^{0 \cdot e_{0}+1 \cdot e_{1}+2 \cdot e_{2}+\cdots+(k-1) e_{k-1}}}=\frac{\gamma}{m^{k}} \quad \text { with } \gamma \in R
$$

Hence $\Delta_{m, k}\left(c_{0}, \ldots, c_{k-1}\right) \in m^{-k} R$.

Next, since $k \geq 1$ and $b_{k} \in R$, we see that $k ! b_{k} / m \in m^{-k} R$. Finally, we note that $c_{\ell} \cdot \mathbb{Z}\left[b_{1}, b_{2}, \ldots\right] \subset m^{-\ell} R$ with $\ell \leq k / m<k$, so these terms are also in $m^{-k} R$. This concludes the proof by induction that $c_{k} \in m^{-k} R$ for all $k \geq 1$.

This completes the proof that the inverse Böttcher coordinate $f_{\varphi}^{-1}(x)$ has the desired form. To complete the proof of Theorem 3 , we need merely note that Proposition 15(c,d) then tells us that $f_{\varphi}(x)$ also has the desired form.

\section{Proof of Theorem 5}

Proof of Theorem 5. We will make frequent use of Legendre's formula for the valuation of a factorial. Writing the base- $p$ expansion of a nonnegative integer $N$ as

$$
N=\sum_{i \geq 0} N_{i} p^{i} \quad \text { with } \quad 0 \leq N_{i}<p, \quad \text { we let } \quad S_{p}(N):=\sum_{i \geq 0} N_{i} .
$$

Then Legendre's formula says that

$$
\operatorname{ord}_{p}(N !)=\frac{N-S_{p}(N)}{p-1} .
$$

In particular,

$$
N \text { is a power of } p \Longrightarrow \operatorname{ord}_{p}(N !)=\frac{N-1}{p-1} .
$$

We note that $a_{0}=1$, and one easily checks that $a_{1}=-1$. We are going to prove that if $k$ is a non-trivial power of $p$, then

$$
a_{k} \equiv a_{k / p}(\bmod p) \text {. }
$$

Combined with the initial value $a_{1}=-1$, this clearly implies that $a_{k} \equiv-1(\bmod p)$ for all $k$ that are powers of $p$. 
So we take $k$ to be a power of $p$ with $k \geq p$. To ease notation (and with a view to generalizations), we let

$$
q:=p^{2}
$$

Expanding the Böttcher equation $\varphi\left(f_{\varphi}(x)\right)=f\left(x^{q}\right)$, we find that the coefficient of $x^{k}$ gives us a recursive formula for $a_{k}$ in terms of the lower $a_{i}$. More specifically, we can write $a_{k}$ as a sum of three terms

$$
a_{k}=A_{k}\left[x^{k}\right]-B_{k}\left[x^{k}\right]-C_{k}\left[x^{k}\right]
$$

where as in (6) , we write $P\left[x^{k}\right]$ for the coefficient of $x^{k}$ in a polynomial or power series $P(x)$, and where $A_{k}, B_{k}$, and $C_{k}$ are given by the formulas

$$
A_{k}:=\frac{k !}{q} \sum_{\ell=0}^{k-1} \frac{a_{\ell}}{\ell !} x^{q \ell}, \quad B_{k}:=\frac{k !}{q}\left(\sum_{\ell=0}^{k-1} \frac{a_{\ell}}{\ell !} x^{\ell}\right)^{q}, C_{k}:=k !\left(x \sum_{\ell=0}^{k-1} \frac{a_{\ell}}{\ell !} x^{\ell}\right)^{q+1} .
$$

We start by analyzing $B_{k}\left[x^{k}\right]$, since this is the term in which we will find a single monomial in the $a_{i}$ whose coefficient is prime to $p$. Expanding the $q$ 'th power that defines $B_{k}$ yields

$$
\begin{aligned}
& B_{k}\left[x^{k}\right]=\frac{k !}{q} \sum_{\begin{array}{c}
e_{0}+e_{1}+\cdots+e_{k-1}=q \\
0 \cdot e_{0}+1 \cdot e_{1}+\cdots+(k-1) e_{k-1}=k
\end{array}}\left(\begin{array}{c}
q \\
e_{0}, e_{1}, \ldots, e_{k-1}
\end{array}\right) \\
& \cdot\left(\frac{a_{0}}{0 !}\right)^{e_{0}}\left(\frac{a_{1}}{1 !}\right)^{e_{1}}\left(\frac{a_{2}}{2 !}\right)^{e_{2}} \cdots\left(\frac{a_{k-1}}{(k-1) !}\right)^{e_{k-1}} .
\end{aligned}
$$

For any given $\boldsymbol{e}:=\left(e_{0}, \ldots, e_{k-1}\right)$, the coefficent of $\boldsymbol{a}^{\boldsymbol{e}}:=a_{0}^{e_{0}} \cdots a_{k-1}^{e_{k-1}}$ in $B_{k}\left[x^{k}\right]$ is

$$
B_{k}\left[x^{k}\right]\left[\boldsymbol{a}^{e}\right]:=\frac{k !}{q} \cdot\left(\begin{array}{c}
q \\
e_{0}, e_{1}, \ldots, e_{k-1}
\end{array}\right) \cdot \prod_{n=0}^{k-1} \frac{1}{n ! e_{n}} .
$$

We claim that

$$
\begin{aligned}
& \operatorname{ord}_{p} B_{k}\left[x^{k}\right]\left[\boldsymbol{a}^{\boldsymbol{e}}\right]=0 \quad \text { if } \boldsymbol{a}^{\boldsymbol{e}}=a_{0}^{q-p} a_{k / p}^{p}, \\
& \operatorname{ord}_{p} B_{k}\left[x^{k}\right]\left[\boldsymbol{a}^{\boldsymbol{e}}\right]>0 \quad \text { otherwise. }
\end{aligned}
$$

In (17) we expressed $B_{k}\left[x^{k}\right]\left[\boldsymbol{a}^{\boldsymbol{e}}\right]$ as a product of three terms. We compute the valuations of these three terms, using the fact that $k$ and $q$ are powers of $p$, and using Legendre's formula (14) to compute the valuations of various factorials. Thus

$$
\operatorname{ord}_{p}\left(\frac{k !}{q}\right)=\frac{k-1}{p-1}-\operatorname{ord}_{p}(q)
$$




$$
\begin{aligned}
& \operatorname{ord}_{p}\left(\begin{array}{c}
q \\
e_{0}, e_{1}, \ldots, e_{k-1}
\end{array}\right)=\operatorname{ord}_{p}(q !)-\sum_{n=0}^{k-1} \operatorname{ord}_{p}\left(e_{n} !\right) \\
&=\frac{1}{p-1}\left((q-1)-\sum_{n=0}^{k-1}\left(e_{n}-S_{p}\left(e_{n}\right)\right)\right) \\
&=\frac{1}{p-1}\left(-1+\sum_{n=0}^{k-1} S_{p}\left(e_{n}\right)\right) \\
& \operatorname{ord}_{p}\left(\prod_{n=0}^{k-1} \frac{1}{n ! e_{n}}\right)=-\frac{1}{p-1} \sum_{n=0}^{k-1} e_{n}\left(n-S_{p}(n)\right) \\
&=\frac{1}{p-1}\left(-k+\sum_{n=0}^{k-1} e_{n} S_{p}(n)\right) \\
& \\
& \operatorname{cosing}_{n} \sum n e_{n}=k .
\end{aligned}
$$

Adding these three pieces and using $\operatorname{ord}_{p}(q)=2$, we find that

$$
(p-1) \operatorname{ord}_{p} B_{k}\left[x^{k}\right]\left[\boldsymbol{a}^{\boldsymbol{e}}\right]=-2 p+\sum_{n=0}^{k-1}\left(e_{n} S_{p}(n)+S_{p}\left(e_{n}\right)\right) .
$$

As a warm-up, we use (20) to prove (18), which is one of our claims. Thus

$$
\begin{aligned}
(p-1) \operatorname{ord}_{p} B_{k}\left[x^{k}\right]\left[a_{0}^{q-p} a_{k / p}^{p}\right] & =-2 p+S_{p}\left(p^{2}-p\right)+p S_{p}(k / p)+S_{p}(p) \\
& =-2 p+(p-1)+p+1=0 .
\end{aligned}
$$

Before proceeding, we are going to rewrite (20) to exploit the fact that $S_{p}(n) \geq 1$ for all $n \geq 1$. So we pull the $n=0$ term out of the sum, replace $S_{p}(n)$ with $S_{p}(n)-1+1$, and use the fact that $\sum e_{n}=q=p^{2}$. This yields

$$
\begin{aligned}
& (p-1) \operatorname{ord}_{p} B_{k}\left[x^{k}\right]\left[\boldsymbol{a}^{e}\right] \\
& \quad=p^{2}-2 p-e_{0}+S_{p}\left(e_{0}\right)+\sum_{n=1}^{k-1}\left(e_{n}\left(S_{p}(n)-1\right)+S_{p}\left(e_{n}\right)\right) .
\end{aligned}
$$

Hence

$$
\begin{aligned}
\operatorname{ord}_{p} B_{k}\left[x^{k}\right]\left[\boldsymbol{a}^{e}\right] \leq 0 & \\
& \Longleftrightarrow \sum_{n=1}^{k-1}\left(e_{n}\left(S_{p}(n)-1\right)+S_{p}\left(e_{n}\right)\right) \leq e_{0}-p^{2}+2 p-S_{p}\left(e_{0}\right) .
\end{aligned}
$$


In particular, we have $e_{0} \geq p^{2}-2 p$, while $\sum e_{n}=p^{2}$ combined with $\sum n e_{n}=k>0$ tell us that $e_{0}<p^{2}$. Thus $p^{2}-2 p \leq e_{0}<p^{2}$. We split this interval into two pieces. Thus for $0 \leq j<p$, we have

\begin{tabular}{|c|c|c|}
\hline$e_{0}$ & $S_{p}\left(e_{0}\right)$ & $e_{0}-p^{2}+2 p-S_{p}\left(e_{0}\right)$ \\
\hline \hline$p^{2}-2 p+j$ & $p-2+j$ & $2-p$ \\
\hline$p^{2}-p+j$ & $p-1+j$ & 1 \\
\hline
\end{tabular}

Since $p \geq 2$, we have proven that

$$
\begin{aligned}
& \operatorname{ord}_{p} B_{k}\left[x^{k}\right]\left[\boldsymbol{a}^{e}\right] \leq 0 \\
& \Longrightarrow \sum_{n=1}^{k-1}\left(e_{n}\left(S_{p}(n)-1\right)+S_{p}\left(e_{n}\right)\right) \leq \begin{cases}0 & \text { if } p^{2}-2 p \leq e_{0}<p^{2}-p, \\
1 & \text { if } p^{2}-p \leq e_{0}<p^{2}\end{cases}
\end{aligned}
$$

We know from $\sum n e_{n}=k$ that there exists at least one $m \geq 1$ such that $e_{m} \geq 1$, and for each such $m$ we have $S_{p}\left(e_{m}\right) \geq 1$. Thus there is a unique $m \geq 1$ with $e_{m} \geq 1$. Further, we observe that if $e_{m}$ is not a power of $p$, then $S_{p}\left(e_{m}\right) \geq 2$, so we conclude that $e_{m}$ is a power of $p$. Also, since the sum is strictly positive, we see that $p^{2}>e_{0} \geq p^{2}-p$. We now know the following three facts:
(1) $p^{2}=e_{0}+e_{m}$.
(2) $p^{2}>e_{0} \geq p^{2}-p$.
(3) $e_{m}$ is a power of $p$.

Thus $e_{m}$ is a power of $p$ satisfying $p \geq e_{m}>0$, which proves that $e_{m}=$ $p$. Then

$$
k=\sum_{n=0}^{k-1} n e_{n}=m e_{m} \quad \Longrightarrow \quad m=k / e_{m}=k / p .
$$

This proves that

$$
\operatorname{ord}_{p} B_{k}\left[x^{k}\right]\left[\boldsymbol{a}^{\boldsymbol{e}}\right] \leq 0 \quad \Longrightarrow \quad \boldsymbol{a}^{\boldsymbol{e}}=a_{0}^{q-p} a_{k / p}^{p},
$$

which concludes the proof of (19).

We next consider $A_{k}$. For $k$ a power of $p$, we have

$$
A_{k}\left[x^{k}\right]= \begin{cases}0 & \text { if } k<q, \\ \frac{k ! a_{k / q}}{q(k / q) !} & \text { if } k \geq q .\end{cases}
$$

For $k \geq q=p^{2}$ we compute

$$
\begin{aligned}
\operatorname{ord}_{p}\left(\frac{k !}{q(k / q) !}\right) & =\frac{k-1}{p-1}-2-\frac{k / q-1}{p-1} \\
& =(p-1)\left(\frac{k}{q}(p+1)-2\right)>(p-1)^{2}>0 .
\end{aligned}
$$


Hence

$$
A_{k}\left[x^{k}\right] \equiv 0 \quad(\bmod p) .
$$

We next consider $C_{k}\left[x^{k}\right]$. Expanding the power, and noting that there is an $x^{q+1}$ that comes out, we find a formula similar to formula (16) for $B_{k}\left[x^{k}\right]$, the primary difference being that there is no $1 / q$ and the sum is over a different collection of indices. With the obvious notation for the multinomial coefficient, we have

$$
C_{k}\left[x^{k}\right]=k ! \sum_{\substack{e_{0}+e_{1}+\cdots+e_{k-1}=q+1 \\
0 \cdot e_{0}+1 \cdot e_{1}+\cdots+(k-1) e_{k-1}=k-q-1}}\left(\begin{array}{l}
q \\
\boldsymbol{e}
\end{array}\right) \prod_{n=0}^{k-1}\left(\frac{a_{n}}{n !}\right)^{e_{n}} .
$$

For any given $\boldsymbol{e}:=\left(e_{0}, \ldots, e_{k-1}\right)$, the coefficent of $\boldsymbol{a}^{\boldsymbol{e}}:=a_{0}^{e_{0}} \cdots a_{k-1}^{e_{k-1}}$ in $C_{k}\left[x^{k}\right]$ is

$$
C_{k}\left[x^{k}\right]\left[\boldsymbol{a}^{e}\right]=k ! \cdot\left(\begin{array}{c}
q \\
e_{0}, e_{1}, \ldots, e_{k-1}
\end{array}\right) \cdot \prod_{n=0}^{k-1} \frac{1}{n !^{e_{n}}} .
$$

We claim that $C_{k}\left[x^{k}\right]\left[\boldsymbol{a}^{\boldsymbol{e}}\right]$ is always divisible by $p$. To see this, we compute

$$
\begin{aligned}
& (p-1) \operatorname{ord}_{p} C_{k}\left[x^{k}\right]\left[\boldsymbol{a}^{e}\right] \\
& \quad=(k-1)+(q-1)-\sum_{n=0}^{k-1}\left(e_{n}-S_{p}\left(e_{n}\right)\right)-\sum_{n=0}^{k-1} e_{n}\left(n-S_{p}(n)\right) \\
& \quad=(k-1)+(q-1)-(q+1)-(k-q-1)+\sum_{n=0}^{k-1}\left(S_{p}\left(e_{n}\right)+e_{n} S_{p}(n)\right) \\
& \quad=q-2+\sum_{n=0}^{k-1}\left(S_{p}\left(e_{n}\right)+e_{n} S_{p}(n)\right) .
\end{aligned}
$$

Since $q=p^{2} \geq 4$, this quantity is certainly positive.

Combining our computations for $A_{k}\left[x^{k}\right], B_{k}\left[x^{k}\right]$, and $C_{k}\left[x^{k}\right]$, we have proven that for $k$ a power of $p$, the recursion (15) for $a_{k}$ in terms of $a_{i}$ with $i<k$ has the following form:

$$
a_{k} \equiv-\frac{k !}{q} \cdot\left(\begin{array}{c}
q \\
q-p, p
\end{array}\right) \cdot\left(\frac{a_{0}}{0 !}\right)^{q-p} \cdot\left(\frac{a_{k / p}}{(k / p) !}\right)^{p} \quad(\bmod p) .
$$

In order to simplify (22), we set a useful piece of notation. For $N \geq 1$, we let

$$
T_{p}(N):=\text { prime-to- } p \text { part of } N=N / p^{\operatorname{ord}_{p}(N)} .
$$


Applying $T_{p}$ to (22) yields

$$
\frac{a_{k}}{a_{k / p}^{p}}=-\frac{T_{p}(k !) T_{p}(q !)}{T_{p}((q-p) !) T_{p}(p !) T_{p}((k / p) !)^{p}},
$$

and since we are only interested in the value of $T_{p}\left(a_{k}\right) \bmod p$, we can use Fermat's little theorem to simplify this to

$$
\frac{a_{k}}{a_{k / p}} \equiv-\frac{T_{p}(k !) T_{p}(q !)}{T_{p}((q-p) !) T_{p}(p !) T_{p}((k / p) !)} \quad(\bmod p) .
$$

It remains to compute the congruence class of the right-hand side of (23), for which we use the following elementary lemma.

Lemma 20. For all primes $p$ and all $r \geq 0$, we have

$$
T_{p}\left(\left(p^{r}\right) !\right) \equiv(-1)^{r} \quad(\bmod p) .
$$

Proof. We may assume that $p$ is odd. We group the numbers from 1 to $p^{r}$ by their $p$-adic valuation. This yields

$$
\begin{aligned}
T_{p}\left(\left(p^{r}\right) !\right) & =\prod_{j=0}^{r-1} \prod_{\substack{1 \leq n<p^{r-j} \\
p \nmid n}} T_{p}\left(p^{j} n\right)=\prod_{j=0}^{r-1} \prod_{\substack{1 \leq n<p^{r-j} \\
p \nmid n}} n \\
& \equiv \prod_{j=0}^{r-1}((p-1) !)^{p^{r-j-1}} \quad(\bmod p) \\
& \equiv \prod_{j=0}^{r-1}(-1)^{p^{r-j-1}} \quad(\bmod p) \quad \text { Wilson's theorem, } \\
& \equiv(-1)^{r} \quad(\bmod p) .
\end{aligned}
$$

This completes the proof of Lemma 20.

Lemma 20 allows us to evaluate every factor in (23) except $(q-p)$ !. For that term, we use the lemma with $q=p^{2}$ and Wilson's theorem to compute

$$
\begin{aligned}
T_{p}((q-p) !) & =T_{p}\left(\frac{q !}{q(q-1) \cdots(q-(p-1))}\right) \\
& \equiv \frac{(-1)^{2}}{(-1)^{p-1}(p-1) !} \equiv-1 \quad(\bmod p) .
\end{aligned}
$$

Writing $k=p^{r}$ and using $q=p^{2}$, we use Lemma 20 and (24) to compute the right-hand side of (23):

$$
\frac{a_{k}}{a_{k / p}} \equiv-\frac{T_{p}\left(p^{r} !\right) T_{p}\left(p^{2} !\right)}{T_{p}\left(\left(p^{2}-p\right) !\right) T_{p}(p !) T_{p}\left(p^{r-1} !\right)} \quad(\bmod p)
$$




$$
\equiv-\frac{(-1)^{r}(-1)^{2}}{(-1)(-1)(-1)^{r-1}} \equiv 1 \quad(\bmod p) .
$$

This completes the proof that $a_{k} \equiv a_{k / p}(\bmod p)$, and hence the proof of Theorem 5.

Remark 21. During the proof of Theorem 5, we derived a formula (20) for the quantity $\operatorname{ord}_{p} B_{k}\left[x^{k}\right]\left[\boldsymbol{a}^{\boldsymbol{e}}\right]$ under the assumption that $q=p^{2}$ and $k$ is a power of $p$. We note that this calculation easily generalizes for arbitrary $q$ and $k$. Without giving the details, we list the result in the hope that it might be useful in future investigations, e.g., in proving Conjecture 23:

$$
\begin{aligned}
(p-1) \operatorname{ord}_{p} B_{k}\left[x^{k}\right]\left[\boldsymbol{a}^{e}\right]=q & -e_{0}-(p-1) \operatorname{ord}_{p}(q)-S_{p}(k)-S_{p}(q) \\
& +S_{p}\left(e_{0}\right)+\sum_{n=1}^{k-1}\left(e_{n}\left(S_{p}(n)-1\right)+S_{p}\left(e_{n}\right)\right) .
\end{aligned}
$$

\section{The RAdius of CONVERGENCE OF BÖTtChER COORDINATES}

In this section we use Theorems 3, 4, and 5 to prove Corollary 6, which describes the radius of convergence of the Böttcher coordinate of various sorts of power series.

We start with an elementary lemma that is undoubtedly well-known, but for lack of a suitable reference and for the convenience of the reader, we give the short proof. We set the notation

$$
\mathcal{D}(R):=\left\{x \in \mathbb{C}_{p}:\|x\|_{p}<R\right\} .
$$

Lemma 22. (a) Let

$f(x)=x \sum_{k=0}^{\infty} a_{k} x^{k} \in \mathbb{C}_{p} \llbracket x \rrbracket$ with $\left\|a_{0}\right\|_{p}=1$ and all $\left\|a_{k}\right\|_{p} \leq 1$.

Then $f(x)$ and its inverse converge and induce an isometry

$$
f: \mathcal{D}(1) \stackrel{\sim}{\longrightarrow} \mathcal{D}(1) .
$$

(b) Let

$$
f(x)=x \sum_{k=0}^{\infty} \frac{a_{k} x^{k}}{k !} \in \mathbb{C}_{p} \llbracket x \rrbracket \text { with }\left\|a_{0}\right\|_{p}=1 \text { and all }\left\|a_{k}\right\|_{p} \leq 1 .
$$

Then $f(x)$ and its inverse converge and induce an isometry

$$
f: \mathcal{D}\left(p^{-1 /(p-1)}\right) \stackrel{\sim}{\longrightarrow} \mathcal{D}\left(p^{-1 /(p-1)}\right) .
$$


Proof. We give the proof of (b), since the proof of (a) is similar, but easier. The fact that $f(x)$ converges on $\mathcal{D}\left(p^{-1 /(p-1)}\right)$ is standard and follows from the estimate

$$
\begin{aligned}
\limsup _{k \rightarrow \infty}\left\|\frac{a_{k}}{k !}\right\|_{p}^{-\frac{1}{k+1}} \geq \limsup _{k \rightarrow \infty}\|k !\|_{p}^{-\frac{1}{k+1}} & \\
\quad & =\limsup _{k \rightarrow \infty}\left(p^{-\frac{k-S_{p}(k)}{p-1}}\right)^{-\frac{1}{k+1}}=p^{-\frac{1}{p-1}} .
\end{aligned}
$$

For the last equality, we used $S_{p}(k) \leq(p-1) \log (k) / \log (p)$, valid for all $k \geq 1$.

In order to show that $f$ is an isometry, we note that

$$
\frac{f(x)-f(y)}{x-y}=a_{0}+\sum_{k=1}^{\infty} \frac{a_{k}}{k !} \cdot \frac{x^{k+1}-y^{k+1}}{x-y} .
$$

Since $\left\|a_{0}\right\|_{p}=1$ by assumption, we need to show that every term in the sum has norm strictly smaller than 1 . We first observe that for $k \geq 0$ and (distinct) $x, y \in \mathcal{D}$, we have

$$
\left\|\frac{x^{k+1}-y^{k+1}}{x-y}\right\|_{p}=\left\|\sum_{i=0}^{k} x^{i} y^{k-i}\right\|_{p} \leq \max _{0 \leq i \leq k}\left\|x^{i} y^{k-i}\right\|_{p} \leq p^{-\frac{k}{p-1}} .
$$

Hence using $\left\|a_{k}\right\|_{p} \leq 1$ and the fact that $S_{p}(k) \geq 1$ for all $k \geq 1$, we find that

$$
\begin{aligned}
\sup _{k \geq 1}\left\|\frac{a_{k}}{k !} \cdot \frac{x^{k+1}-y^{k+1}}{x-y}\right\|_{p} & \leq \sup _{k \geq 1}\left\|\frac{1}{k !}\right\|_{p} \cdot p^{-\frac{k}{p-1}} \quad \text { from (25) }, \\
& =\sup _{k \geq 1} p^{\frac{k-S_{p}(k)}{p-1}} \cdot p^{-\frac{k}{p-1}} \\
& =\sup _{k \geq 1} p^{-\frac{S_{p}(k)}{p-1}}=p^{-\frac{1}{p-1}}<1 .
\end{aligned}
$$

This concludes the proof of (b).

Proof of Corollary 6. Proposition 2 and Lemma 22(a) immediately imply the part of Corollary 6(a) with $p \nmid m$, while Theorem 4 and Lemma 22(a) immediately imply Corollary 6(c).

Similarly, Theorem 3(b) and Lemma 22(b) immediately imply Corollary 6(b).

Next, to prove the $p \mid m$ part of Corollary 6(a), we use Theorem 3(a) and apply Lemma22(b) to the series $m f_{\varphi}(x / m)$. Undoing this transformation transforms the disk $\mathcal{D}\left(p^{-1 /(p-1)}\right)$ to the disk $\mathcal{D}\left(p^{-1 /(p-1)}\|m\|_{p}\right)$. 
Finally, to prove $(\mathrm{d})$, we use Theorem 5 to compute the radius of convergence of $f_{\varphi}(x)$. Thus

$$
\begin{aligned}
\rho\left(f_{\varphi}\right): & =\liminf _{k \rightarrow \infty}\left\|a_{k} / k !\right\|_{p}^{-1 /(k+1)} \\
= & \liminf _{k \rightarrow \infty} p^{\frac{\operatorname{ord}\left(a_{k}\right)-\operatorname{ord} p(k !)}{k+1}} \\
= & \liminf _{k \rightarrow \infty} p^{\left(\operatorname{ord}_{p}\left(a_{k}\right)-\frac{k-S_{p}(k)}{p-1}\right) \cdot \frac{1}{k+1}} \\
= & p^{-1 /(p-1)} \cdot \liminf _{k \rightarrow \infty} p^{\operatorname{ord}_{p}\left(a_{k}\right) /(k+1)} \\
& \quad \text { ince } S_{p}(k) \leq(p-1) \log _{p}(k+1), \text { so } S_{p}(k) /(k+1) \rightarrow 0, \\
= & p^{-1 /(p-1)} \quad \text { since Theorem 5 says } a_{k}=-1 \text { for infinitely many } k .
\end{aligned}
$$

This completes the proof of Corollary 6 .

\section{The BötTCher COORdinate OF $x^{p^{2}}+p^{r} x^{p^{2}+1}$}

This section describes a number of conjectures prompted by moderately extensive numerical experiments.

Theorem 5 says that the $k$ 'th coefficient $a_{k} / k$ ! of the Böttcher coordinate of $x^{p^{2}}+p^{2} x^{p^{2}+1}$ satisfies $a_{k} \equiv-1(\bmod p)$ whenever $k$ is a power of $p$. Experiments suggest that the sequence of $\bmod p$ values of the $a_{k}$ have many interesting properties. Computations for primes $2 \leq p \leq 11$ and $0 \leq k \leq 50$ lead us to make the following conjecture.

Conjecture 23. Let $\varphi(x)=x^{p^{2}}+p^{2} x^{p^{2}+1}$, and write the Bötcher coordinate of $\varphi$ as $f_{\varphi}(x)=x \sum_{k=0}^{\infty}\left(a_{k} / k !\right) x^{k}$, so Theorem $5(b)$ tells us that the $a_{k}$ are integers. Then

$$
\begin{aligned}
& k \equiv 0(\bmod p) \quad \Longrightarrow \quad a_{k} \equiv(-1)^{k / p}(\bmod p), \\
& k \equiv-2(\bmod p) \quad \Longrightarrow \quad a_{k} \equiv-1(\bmod p) \text {, } \\
& k \equiv-1(\bmod p) \quad \Longleftrightarrow \quad a_{k} \equiv 0(\bmod p) \text {, }
\end{aligned}
$$

with the one exception that for $p=2$, we have $a_{1} \equiv 1(\bmod 2)$.

Remark 24. Let $B_{k}\left[x^{k}\right]$ be as in the proof of Theorem 5. The key to the proof of that theorem is that when $k$ is a power of $p$, the expression for $B_{k}\left[x^{k}\right]$ as a polynomial in $a_{0}, \ldots, a_{k-1}$ contains a single monomial whose coefficient is not divisible by $p$. Precisely, we proved that

$$
-B_{k}\left[x^{k}\right] \equiv a_{0}^{p^{2}-p} a_{k / p}^{p}(\bmod p) .
$$


One approach to the first part of Conjecture 23 might be to show that $B_{k}\left[x^{k}\right] \bmod p$ consists of a single monomial under the weaker assumption that $p \mid k$. Unfortunately, this is not true. To illustrate, we list the first few cases for which it fails for $p=2$ :

$$
\begin{aligned}
B_{14}\left[x^{14}\right] \bmod 2 & =a_{0}^{2}\left(a_{2} a_{12}+a_{4} a_{10}+a_{6} a_{8}\right), \\
B_{22}\left[x^{22}\right] \bmod 2 & =a_{0}^{2}\left(a_{2} a_{20}+a_{4} a_{18}+a_{6} a_{16}\right), \\
B_{26}\left[x^{26}\right] \bmod 2 & =a_{0}^{2}\left(a_{2} a_{24}+a_{8} a_{18}+a_{10} a_{16}\right), \\
B_{28}\left[x^{28}\right] \bmod 2 & =a_{0}^{2}\left(a_{4} a_{24}+a_{8} a_{20}+a_{12} a_{16}\right), \\
B_{30}\left[x^{30}\right] \bmod 2 & =a_{0}^{2}\left(a_{2} a_{28}+a_{4} a_{26}+a_{6} a_{24}+a_{8} a_{22}\right. \\
& \left.+a_{10} a_{20}+a_{12} a_{18}+a_{14} a_{16}\right) .
\end{aligned}
$$

We observe that in each case the sum consists of an odd number of monomials, each of which is a product of even index $a_{i}$, which is consistent with the conjecture that $B_{k}\left[x^{k}\right]$ is odd when $k$ is even.

We next consider the coefficients for the Böttcher coordinate of

$$
\varphi(x)=x^{p^{2}}+p^{r} x^{p^{2}+1}
$$

as $r$ increases. Not surprisingly, the integrality of the coefficients of the Böttcher coordinate increases with increasing $r$, but we conjecture that we never achieve a Böttcher coordinate whose coefficients are entirely $p$ integral.

More precisely, experiments suggest that the valuations of the Böttcher quantities $a_{k}$ for $p \mid k$ exhibit a great deal of regularity. Here is a typical example. For the function

$$
\varphi(x)=x^{4}+2^{8} x^{5},
$$

we observe numerically that that the coefficients of the Böttcher coordinate $f_{\varphi}(x)=x \sum\left(a_{k} / k !\right) x^{k}$ satisfy the following somewhat complicated recursion:

$$
\begin{aligned}
& \operatorname{ord}_{2}\left(a_{2 n+2}\right)-\operatorname{ord}_{2}\left(a_{2 n}\right) \\
& = \begin{cases}12 & \text { if } n \equiv 0(\bmod 2), \\
-5 & \text { if } n \equiv 1(\bmod 2) \text { and } n \neq-1(\bmod 8), \\
-16 & \text { if } n \equiv-1(\bmod 8) \text { and } n \neq \equiv-1(\bmod 32) . \\
-21 & \text { if } n \equiv-1(\bmod 32) .\end{cases}
\end{aligned}
$$

More generally, for

$$
\varphi(x)=x^{4}+2^{2+r} x^{5} \quad \text { with } r \geq 0,
$$


we find (numerically) that there are recursions for even values of $k$ having the form

$$
\begin{aligned}
\operatorname{ord}_{2}\left(a_{k+2^{r+1}}\right) & =\operatorname{ord}_{2}\left(a_{k}\right)+2^{r+1}-2 & & \text { if } r \text { is odd, } \\
\operatorname{ord}_{2}\left(a_{k+2^{r}}\right) & =\operatorname{ord}_{2}\left(a_{k}\right)+2^{r}-1 & & \text { if } r \text { is even. }
\end{aligned}
$$

Based on these and other computations, we make the following conjecture.

Conjecture 25. Let

$$
\varphi(x)=x^{p^{2}}+p^{2+r} x^{p^{2}+1} \quad \text { with } r \geq 0,
$$

and let $f_{\varphi}(x)=x \sum\left(a_{k} / k !\right) x^{k}$ be the associated Böttcher coordinate.

(a) For $k \equiv 0(\bmod p)$ we have

$$
\operatorname{ord}_{p}\left(a_{k}\right)=\frac{1-p^{-r}}{p-1} k+O(1) \quad \text { as } k \rightarrow \infty .
$$

(b) The radius of convergence of $f_{\varphi}$ is

$$
\rho\left(f_{\varphi}\right):=\liminf _{k \rightarrow \infty}\left\|a_{k} / k !\right\|_{p}^{-1 / k}=p^{-p^{-r} /(p-1)} .
$$

We remark that (b) does not follow from (a). Indeed, applying (a) with $k$ a power of $p$ only shows that the radius of convergence is bounded above by the quantity given by (b).

Acknowledgements. The authors would like to thank Rob Benedetto, Patrick Ingram, Holly Krieger, Jonathan Lubin, and Mike Zieve for their helpful advice, and Melody Chan for her insight into the proof of Lemma 16.

\section{REFERENCES}

[1] J. P. Bell, D. Ghioca, and T. J. Tucker. The dynamical Mordell-Lang problem for étale maps. Amer. J. Math., 132(6):1655-1675, 2010.

[2] L.E.B. Böttcher. The principal laws of convergence of iterates and their application to analysis (Russian). Izv. Kazan.Fiz.-Mat. Obshch., 14:155-234, 1904.

[3] Xavier Buff, Adam L. Epstein, and Sarah Koch. Böttcher coordinates. Indiana Univ. Math. J., 61(5):1765-1799, 2012.

[4] Laura DeMarco, Dragos Ghioca, Holly Krieger, Khoa Nguyen, Thomas Tucker, and Hexi Ye. Bounded height in families of dynamical systems, 2017. arXiv: 1703.05365

[5] D. Ghioca and T. J. Tucker. Periodic points, linearizing maps, and the dynamical Mordell-Lang problem. J. Number Theory, 129(6):1392-1403, 2009.

[6] Patrick Ingram. Arboreal Galois representations and uniformization of polynomial dynamics. Bull. Lond. Math. Soc., 45(2):301-308, 2013.

[7] Warren P. Johnson. The curious history of Faà di Bruno's formula. Amer. Math. Monthly, 109(3):217-234, 2002. 
[8] John Milnor. Dynamics in one complex variable, volume 160 of Annals of Mathematics Studies. Princeton University Press, Princeton, NJ, third edition, 2006.

[9] Steven Roman. The formula of Faà di Bruno. Amer. Math. Monthly, 87(10):805-809, 1980.

[10] Matteo Ruggiero. Classification of one-dimensional superattracting germs in positive characteristic. Ergodic Theory Dynam. Systems, 35(7):2242-2268, 2015.

[11] Joseph H. Silverman. The Arithmetic of Elliptic Curves, volume 106 of Graduate Texts in Mathematics. Springer, Dordrecht, second edition, 2009.

[12] Matthew P. Spencer. Moduli Spaces of Power Series in Finite Characteristic. $\mathrm{PhD}$ thesis, Brown University, 2011.

E-mail address: asalerno@bates.edu

Department of Mathematics, Bates College, Lewiston, Me 04240 USA

E-mail address: jhs@math.brown.edu

Mathematics Department, Box 1917 Brown University, Providence, RI 02912 USA. ORCID: HTTPS://ORCID.ORG/0000-0003-3887-3248 